\title{
SURFACE MODIFICATION OF NANOPARTICLES FOR SUSTAINING SENSITIVITY OF SURFACE-ENHANCED RAMAN SPECTROMETRIC MEASUREMENTS IN SALINATED ENVIRONMENT
}

\author{
${ }^{1}$ Anna TYCOVA, ${ }^{1}$ Jan PRIKRYL, ${ }^{2}$ Petr VANHARA, ${ }^{1}$ Karel KLEPARNIK, ${ }^{1}$ Frantisek FORET \\ ${ }^{1}$ Czech Academy of Sciences, Institute of Analytical Chemistry, Brno, Czech Republic, EU \\ tycova@iach.cz \\ ${ }^{2}$ Department of Histology and Embryology, Faculty of Science, Masaryk University, Brno,
} Czech Republic, EU

https://doi.org/10.37904/nanocon.2019.8480

\begin{abstract}
Surface-enhanced Raman spectrometry (SERS) represents a powerful method for analysis of a broad spectrum of analytes ranging from inorganic ions to biomolecules of high complexity. It combines the potential of Raman spectrometry for a definite identification of an analyte with remarkable sensitivity achieved by the surface enhancement effect occurring on metal nanoparticles. While low ionic strength influences positively the sensitivity of the SERS measurement, a higher level of inorganic salts leads to fast ruining of colloidal character, which completely devastates the effect of the surface enhancement. The common stabilization of nanoparticles by a layer of polymers has a negative impact on the SERS sensitivity since it shields the nanoparticle surface from the analytes. In this work, we aim at the development of the bi-ligand system of nanoparticles surface modification for improved stability of colloid in saline solution at sustaining the potential for sensitive SERS analyses. The proposed system relies on the binding of 3-mercaptopropionic acid and thiolated polyethylene glycol in a suitable ratio onto the nanoparticle surfaces. While the short chains of the acid sustain the accessibility of the surface for analytes, the polymeric structures act as a steric barrier preventing colloid aggregation.
\end{abstract}

Keywords: Bi-ligand, nanoparticle, saline solution, stability, surface-enhanced Raman spectrometry

\section{INTRODUCTION}

From the late 1960s, Raman spectrometry belongs to the methods particularly useful in bulk material characterization. It provides information about the rotational-vibrational states of the analyte, being a key to its definite identification. The Raman spectrum is recorded from inelastically scattered photons, which are (in comparison to elastically interacted photons) very rare. It is estimated that only 1 photon out of $10^{6}-10^{7}$ induces a Raman signal. This hurts the method sensitivity limiting greatly the sphere of potential applications. However, in the 1980s surface enhancement effect was discovered occurring on the nanostructured metal surfaces. [1] Due to the suitable optical properties of nanoscaled silver and gold, they belong to the most commonly used metals.

The effect of the enhancement was, for the first time, observed on a rough silver electrode [1]; however, soon, other platforms were developed usually dealing with precisely organized structures. [2] Their fabrication commonly involves lithographic techniques requiring high-tech equipment and a skilled operator resulting in extensive costs. The use of colloids represents an alternative system for achieving surface enhancement. Their chemical synthesis is simple (reduction of respective salts by a suitable agent) and requires neither any complicated equipment nor extensive costs. Moreover, the nanostructures in the form of dispersion bring much more freedom to experimental design. [3]

SERS provides a particularly strong signal in the spaces called hot-spots, which are formed between the closely located nanostructures. While at solid substrates the hot-spots depend on the surface geometry, in 
colloids they are obtained by controlled aggregation. The aggregation is usually induced by a mild increase in the ionic strength of the sample. Although this can be a very promising strategy in the analysis of model mixtures, it can cause difficulties in the analysis of real-world samples, which often consist of a high level of inorganic ions. This high ionic strength (more than $0.1 \mathrm{M}$ ) causes an extraordinary fast aggregation process leading to the loss of colloidal character (i.e., ruining enhancement effect) within the scale of seconds. [4] Such challenging samples can be found across many areas - medical research (e.g., biological fluids) [5], environmental analysis (e.g., wastewater) or food industry.

The stability of colloids can be achieved by their surface modification, typically by large polymeric structures. Unfortunately, this prevents direct nanoparticle-analyte interaction, resulting in a significant decrease in the sensitivity. [6] In this work, we introduce a feasible system of surface modification of silver nanoparticles (NPs) composed of two ligands, where one of them (long-chained) acts as a steric barrier preventing aggregation, whereas the second one (short-chained) allows close interaction of NP surface with analytes.

\section{EXPERIMENTAL}

\subsection{Chemicals and methods}

The silver colloid was synthesized by a standard Lee-Meisel protocol [7], based on the reduction of silver nitrate (Sigma Aldrich) by sodium citrate (Lachema). The obtained dispersion provided absorption maximum at $420 \mathrm{~nm}$ and an average nanoparticles diameter in the range of 60-70 nm.

For the surface modification of this colloid (Figure 1) mixture of 3-mercaptopropionic acid (MPA) and O-(3Carboxypropyl)-O'-[2-(3-mercaptopropionylamino)ethyl]-polyethylene glycol (3000 Da, PEG-SH) were used, both purchased in Sigma-Aldrich. Both compounds were mixed in deionized water in the desired ratio (for details see the results) to create their mutual solution. This reaction mixture was added into a silver colloid and immediately vortexed for 2 minutes, followed by mild mixing for further 30 minutes. The reagents covalently bound to the nanoparticles via their thiol functional group. The stock solutions of thiolated compounds were prepared freshly every day.

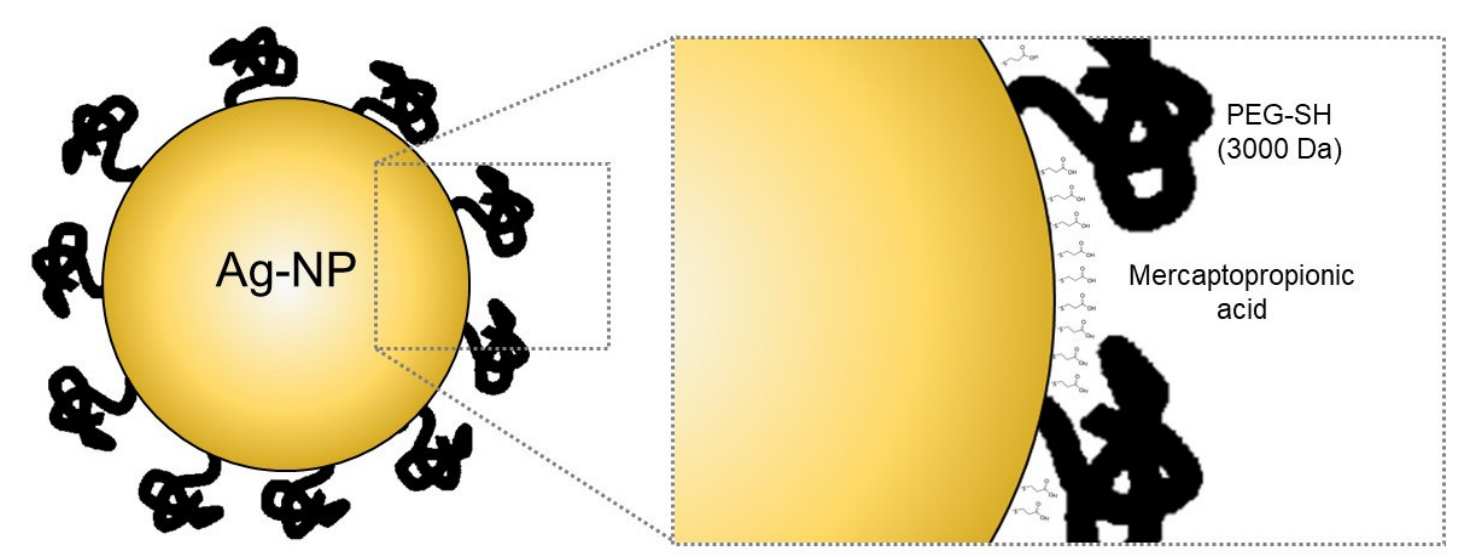

Figure 1 The design of the proposed surface modification of the silver nanoparticles. The figure is not in scale.

\subsection{Instrumentation}

The colloids were characterized using UV-Vis spectrometer (UV-1800, Shimadzu), transmission electron microscopy (FEI Morgagni 268D, Thermo Fisher Scientific), and dynamic light scattering (ZetaSizer Nano ZS, Malvern Instruments). 


\section{RESULTS AND DISCUSSION}

Many real-world samples (e.g., urine, wastewater, food extracts) provide a high content of inorganic salts leading to increased ionic strength. Since the colloidal character of the nanoparticles would be in such an environment devastated within several seconds [4], expensive and complicated strategies are usually employed for SERS analysis of such a sample. [5] An example of the aggregation behavior of the silver colloid is shown in Figure 2, clearly demonstrating the formation of larger entities. This work focuses on the development of a unique system for surface modification of silver nanoparticles, which would lead to reliable and sensitive SERS analyses in salinated samples.

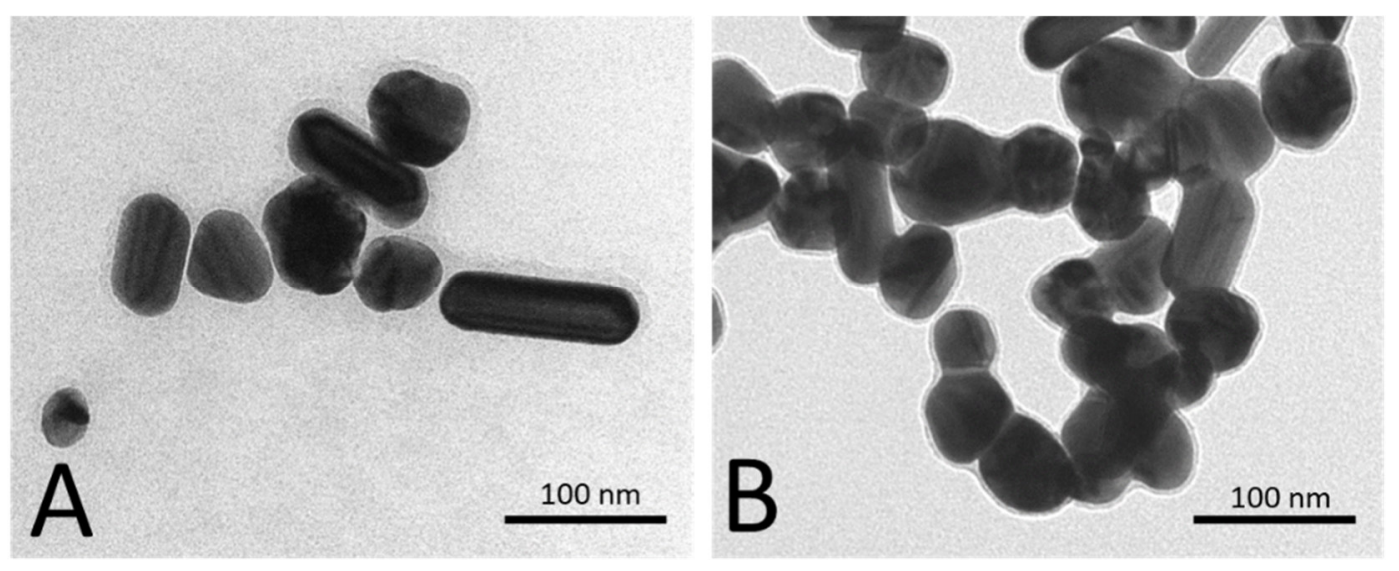

Figure 2 TEM figures of silver nanoparticles:

A - at stable conditions, B - in an aggregated form after the increase of ionic strength

The proposed design is based on the combination of short and long chains of 3-mercaptopropionic acid (MPA) and thiolated polyethylene glycol (PEG-SH), respectively (see Figure 1). At a suitable ratio of these components, areas accessible for the analyte should be formed on the nanoparticle surface (due to the shortchained MPA), while the long-chained polymer should suppress direct NP-NP interactions, thus, preventing their aggregation.

One of the important aspects of the formation of described surface modification is finding a suitable concentration of a modifier, which would ensure complete saturation of the surface of the nanoparticles. Although it is possible to estimate it from theoretical calculations, due to the possible variances in the shape and amount of the nanoparticles (both influencing the surface area), the experiments should bring more relevant data. UV-Vis spectrometry belongs to simple and very effective tools, which can reveal any changes on the nanoparticle surface due to the shifts of the surface plasmon (corresponding to the absorption curve).

Thus, we employed UV-Vis spectrometry to find an optimal concentration of MPA for complete saturation of silver colloid. Figure 3 shows the normalized absorption spectra of six colloids modified by MPA using its various concentrations in the reaction mixture, covering the range of $0.5 \mu \mathrm{M}-250 \mu \mathrm{M}$. Interestingly, concentrations higher than $1.25 \mathrm{mM}$ resulted in rapid colloid aggregation (data not shown). The obtained data nicely showed the shift of absorption maximum from 420 to $424 \mathrm{~nm}$, which can be attributed to the binding of the acid molecules onto the nanoparticles. The saturation was reached at the level of $10 \mu \mathrm{M}$; however, to cover possible batch-to-batch variances in colloid surface capacity, the concentration of $20 \mu \mathrm{M}$ was evaluated as the most appropriate.

The functionality of the proposed bi-ligand design is highly dependent on the suitable ratio of both reagents. While the too high surface concentration of PEG-SH would lead to the blockage of the NPs surface, its shortage would result in a significant drop in the colloid stability, both spoiling the SERS sensitivity. In this work, we tested several molar ratios of MPA and PEG-SH (i.e., 5:1, 10:1, 20:1, 50:1) and modification by PEG-SH only. 
In the reaction mixture, a final concentration of thiolated compounds was always $20 \mu \mathrm{M}$. The modified nanoparticles were introduced into the saline solution, i.e., $0.9 \% \mathrm{NaCl}(\mathrm{w} / \mathrm{v})$, and their UV-Vis absorption spectra were immediately collected. This procedure brought an insight into the stability of colloid in the biological relevant environment. Our observations revealed that the most appropriate molar ratio of MPA:PEG$\mathrm{SH}$ is 10:1 (data not shown). This represented the lowest concentration of PEG-SH in the reaction mixture, at which the unchanged character of UV-Vis spectra was reliably achieved.

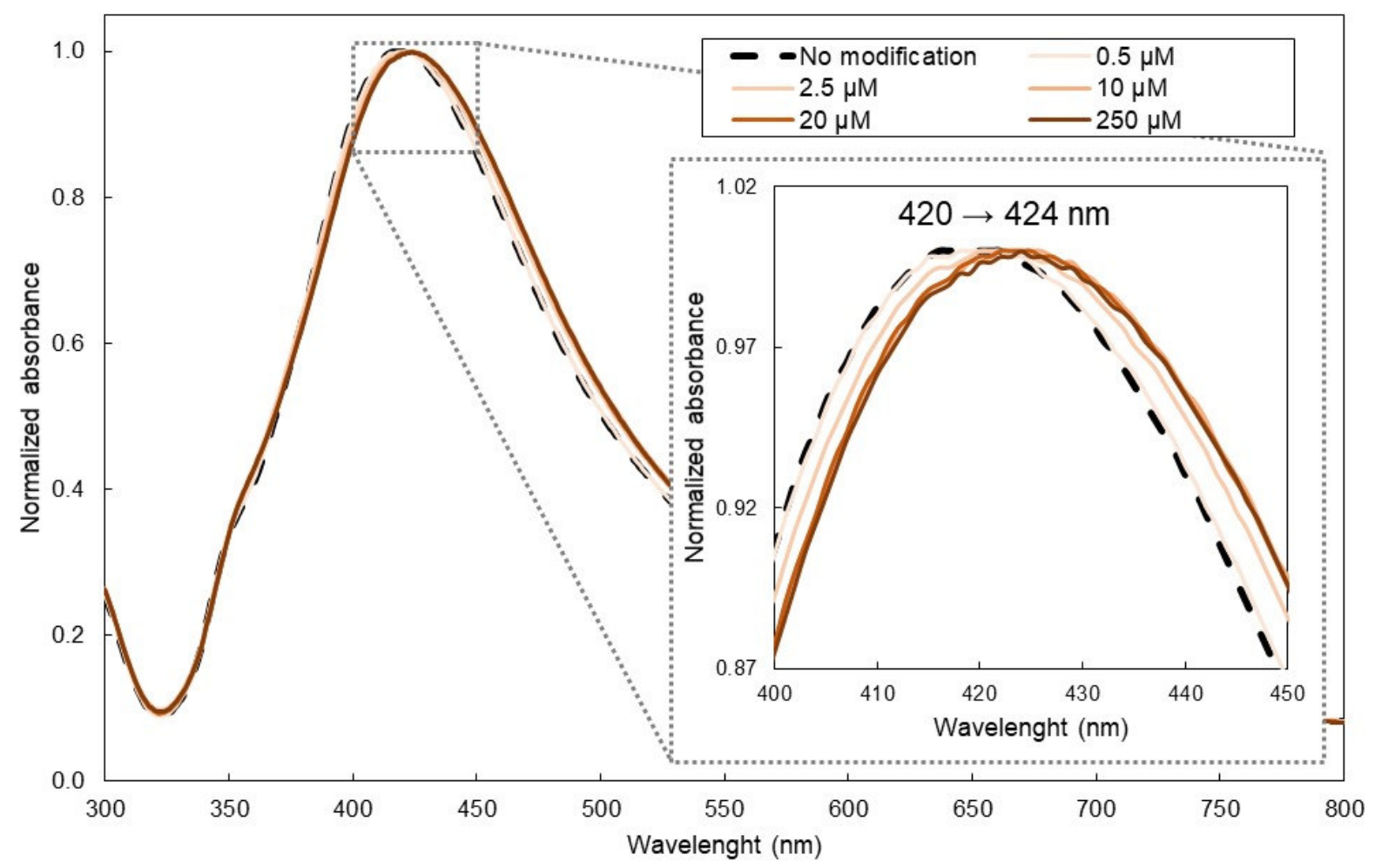

Figure 3 Normalized absorption spectra of silver colloid modified by 3-mercaptopropionic acid (MPA). The legend gives concentration of used MPA.

Our measurements proved the improved stability in salinated solutions; however, it is important to confirm that the conformation of PEG-SH does not block the nanoparticle surface. The conformation of polyethylene glycol is related to its concentration on the nanoparticle surface. At low intermolecular distances, PEG-SH forms socalled brush (molecules interact with each other and are unbent), which would shield the nanostructures from analytes. [8] We aim at a mushroom conformation (depicted in Figure 1), which brings more potential for NPanalyte interaction. Since both conformations differ in PEG-SH size, they affect also the overall nanoparticle diameter. Thus, dynamic light scattering was used for the investigation of an average diameter of two various colloids - modified by PEG-SH only and by MPA:PEG-SH (10:1). A significant change in the dispersion diameters was observed. While the nanoparticles modified by PEG-SH only provided an average diameter of $121 \mathrm{~nm}$, the addition of MPA resulted in its decrease to $107 \mathrm{~nm}$. This proves the formation of the mushroom conformation at the nanoparticle surface.

The aggregation of the silver colloid in the UV-Vis spectra can be observed as a drop of the absorption band at $420 \mathrm{~nm}$, sometimes accompanied by increased absorbance around 600-800 nm. [9] We prepared three batches of colloid modified by MPA:PEG-SH (10:1), MPA (only), and PEG-SH (only) to follow their stability in saline solution within 30 minutes, which provides sufficient time scale for running SERS measurements of the sample. Figure 4 shows the comparison of the UV-Vis spectra taken immediately after the introduction to the 
saline solution and after 30 minutes. Modification by MPA does not have any stabilization effect on the nanoparticles. On the contrary, PEG-SH acts as an excellent stabilizing agent, where no apparent changes were recognized within the followed time. The use of the combination of both agents resulted only in a very mild aggregation and the colloid sustained its character of the dispersion.

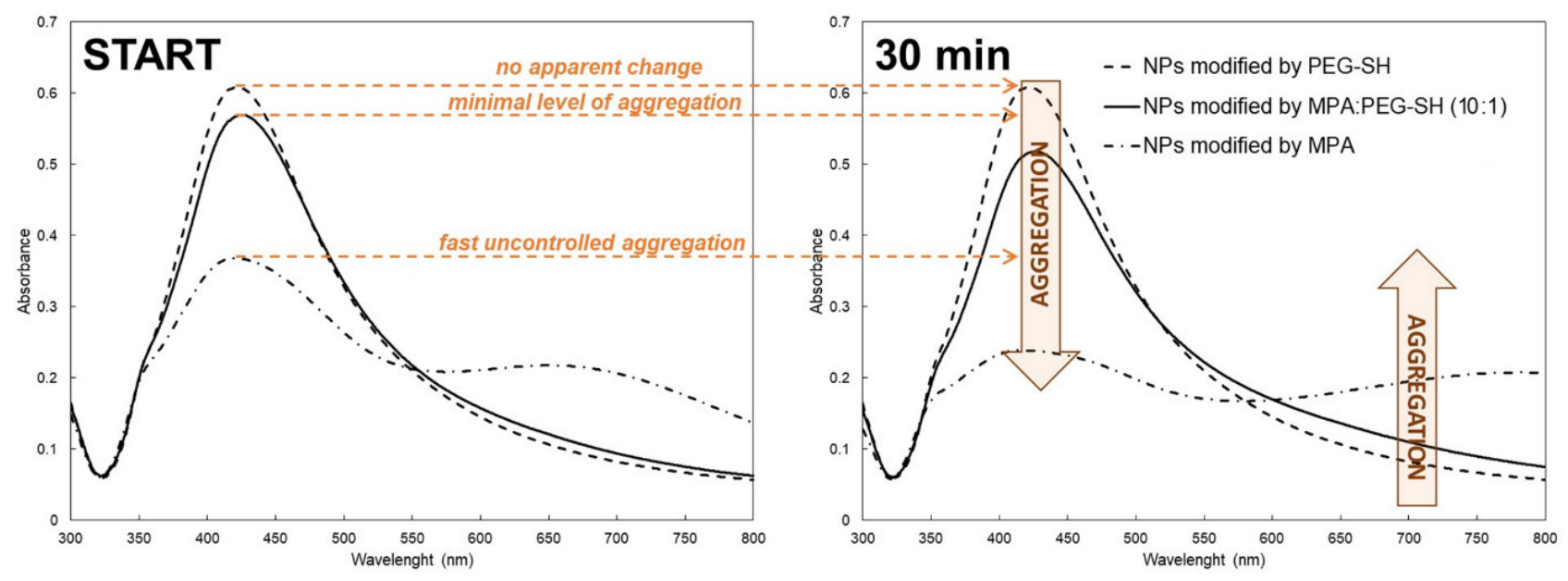

Figure 4 The comparison of the aggregation behavior of three types of modified nanoparticles in a saline solution. Nanoparticles were modified by MPA, PEG-SH, and MPA:PEG-SH (10:1). The final concentration of thiolated compounds in the reaction mixture was always $20 \mu \mathrm{M}$.

\section{CONCLUSION}

In this work, we prepared silver nanoparticles with unique bi-functional surface modification. This modification effectively prevents uncontrolled nanoparticle aggregation (at least for 30 minutes) and has great potential for being a powerful tool for sensitive SERS analyses in solutions of high ionic strength. The design is based on the combination of large polymeric structures and short-chained organic acid. We believe that the adjustment of this short-chained ligand can significantly contribute to the selectivity of the measurement. In the upcoming investigations, these modified nanoparticles will be used for SERS analysis of biomolecules from saline solution to prove their functionality fully. During the SERS measurement, we are going to achieve a favorable aggregation by laser-induced sintering. [10]

\section{REFERENCES}

[1] FLEISCHMANN, Martin, HENDRA, Patrick J. and MCQUILLAN, James A. Raman-Spectra of Pyridine Adsorbed at a Silver Electrode. Chemical Physics Letters. 1974. vol. 26, no. 2, pp. 163-166.

[2] MOSIER-BOSS, Pamela A. Review of SERS Substrates for Chemical Sensing. Nanomaterials. 2017. vol. 7, no. $6,142$.

[3] TYCOVA, Anna, PRIKRYL, Jan and FORET, Frantisek. Recent Strategies toward Microfluidic-Based SurfaceEnhanced Raman Spectroscopy. Electrophoresis. 2017. vol. 38, no. 16, pp. 1977-1987.

[4] PAMIES, Ramon, CIFRE, Jose G. H., FERNANDEZ ESPIN, Vanesa, COLLADO-GONZALEZ, Mar, DIAZ BANOS, Fransisco G. and TORRE, Jose. Aggregation Behaviour of Gold Nanoparticles in Saline Aqueous Media. Journal of Nanoparticle Research. 2014. vol. 16, no. 2376, pp. 1-11.

[5] BONIFACIO, Alois, CERVO, Silvia and SERGO, Valter. Label-Free Surface-Enhanced Raman Spectroscopy of Biofluids: Fundamental Aspects and Diagnostic Applications. Analytical and Bioanalytical Chemistry. 2015, vol. 407, no. 27 , pp. 8265-8277. 
[6] KENNEDY, Brendan J., SPAETH S., DICKEY, Matthew and CARRON, Keith T. Determination of the Distance Dependence and Experimental Effects for Modified SERS Substrates Based on Self-Assembled Monolayers Formed Using Alkanethiols. Journal of Physical Chemistry B. 1999. vol. 103, no. 18, pp. 3640-3646.

[7] LEE, Patrick C. and MEISEL, Dan. Adsorption and Surface-Enhanced Raman of Dyes on Silver and Gold Sols. Journal of Physical Chemistry. 1982. vol. 86, no. 17, pp. 3391-3395.

[8] LABOUTA, Hagar I., GOMEZ-GARCIA, Juliana M., SARSONS, Christopher D., NGUYEN, Trinh, KENNARD, Jacob, NGO, Wayne, TEREFE, Kaisha, IRAGORRI, Nicolas, LAI, Patrick, RINKER, Kristina D. and CRAMB David T. Surface-Grafted Polyethylene Glycol Conformation Impacts the Transport of PEG-Functionalized Liposomes through a Tumour Extracellular Matrix Model. RSC Advances. 2018. vol. 8 , no.14, pp. 7697-7708.

[9] GUERRINI, Luca, GARCIA-RAMOS, Jose V., DOMINGO, Conception and SANCHEZ-CORTES, Santiago. Nanosensors Based on Viologen Functionalized Silver Nanoparticles: Few Molecules Surface-Enhanced Raman Spectroscopy Detection of Polycyclic Aromatic Hydrocarbons in Interparticle Hot Spots. Analytical Chemistry. 2009. vol. 81, no. 4, pp. 1418-1425.

[10] PRIKRYL, Jan, KLEPARNIK, Karel and FORET, Frantisek. Photodeposited Silver Nanoparticles for On-Column Surface-Enhanced Raman Spectrometry Detection in Capillary Electrophoresis. Journal of Chromatography A. 2012. vol. 1226, pp. 43-47. 